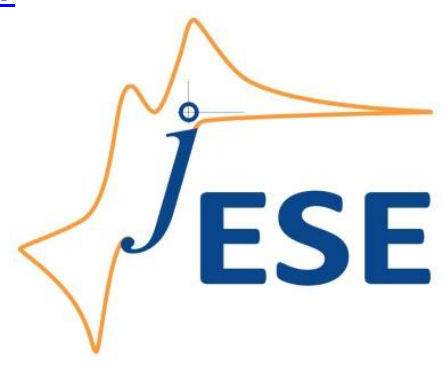

Open Access: ISSN 1847-9286

www.jESE-online.org

Original scientific paper

\title{
A sensitive electrochemical sensor based on polypyrrole/electrochemically reduced graphene oxide for the determination of imidacloprid
}

\author{
Chenglong Chen, Zhen Han, Wu Lei ${ }^{\bowtie}$, Yong Ding, Jingjing Lv, Mingzhu Xia, \\ Fengyun Wang, Qingli Hao ${ }^{\bowtie}$ \\ School of Chemical Engineering, Nanjing University of Science and Technology, \\ Nanjing, 210094, China \\ ${ }^{\otimes}$ Corresponding authors: leiwuhao@njust.edu.cn; Tel.: +86-25-84315943; Fax: +86-25-84315190
}

Received: October 30, 2018; Revised: February 7, 2019; Accepted: February 7, 2019

\begin{abstract}
The glassy carbon electrode (GCE) was modified by electrochemically reduced graphene oxide (ERGO) and polypyrrole (PPy) prepared by simple cyclic voltammetry (CV) electropolymerization. The PPy/ERGO modified electrode (PPy/ERGO/GCE) was used as a platform of electrochemical sensor to detect imidacloprid (IMI) insecticide. CV and differential pulse voltammetry (DPV) were chosen as the methods to investigate of the electrochemical behavior of IMI on PPy/ERGO/GCE surface. Scanning electron microscopy (SEM) and Raman spectra were utilized to describe the morphology and structure of the modified electrode. Experimental parameters were optimized, such as the number of polymerization cycles, scan rate and the $\mathrm{pH}$ value of electrolyte. Under the optimized conditions, when the concentration of $\mathrm{IMI}$ was in the range of 1-10 $\mu \mathrm{M}$ and 10-60 $\mu \mathrm{M}$, the increase of reduction peak current was linear with the concentration of $I \mathrm{MI}$, and the low detection limit was found to be $0.18 \mu \mathrm{M}$ $(S / N=3)$. Results showed that PPy/ERGO/GCE demonstrated satisfactory reproducibility and stability, and has great potential in actual sample testing.
\end{abstract}

\section{Keywords}

Electro-polymerization; modified electrode; imidacloprid insecticide; electrochemical behavior; actual samples

\section{Introduction}

As a widespread used neonicotinoids insecticide, imidacloprid (1-[(6-chloro-3-pyridinyl) methyl]$\mathrm{N}$-nitro-2-imidazolidinimine) has great effective on the pest control by directly acting on the central nervous system of insects [1,2]. Even though IMI is low toxic to mammals, it can move off-site with the underground water and persist as long as several years [3]. The potential contamination and the 
high risk to bees and aquatic invertebrates [4] make the determination of IMI an urgent task since 2013. Among diverse analytical approaches [5-8], the electrochemical method is considered highly efficient, mostly due to its simplicity, sensitivity and feasibility. Most of the reported IMI electrochemical sensors have been focused on the fabrication of the modified electrocatalytic nanomaterials and comprehensive synthesis procedures, such as preparation of gold nanoparticles/paminothiophenol/ $\beta$-cyclodextrin polymer/reduced graphene oxide or imprinted poly/ophenylenediamine) membranes at reduced graphene oxide $[9,10]$. The sensing platform based on the simple electro-polymerization, however, has rarely been mentioned.

Since graphene was firstly reported in 2006 [11], it has attracted great and increasing interest in various areas for its unique mechanical, electronic, thermal properties, large specific surface area and other remarkable characteristics [12]. Graphene oxide (GO) is one of the most attractive derivatives of graphene, which is highly water-soluble and contains large number of oxygencontaining groups (epoxy, carboxyl, hydroxyl, etc.) on its surface and edges [13,14]. Graphene can be chemically reduced from GO via thermal $[15,16]$ or electrochemical [17] reduction, and the product is called reduced graphene oxide (RGO). RGO is also widely used to modify electrodes. It can be used as an electrode modification material alone, or as a component of a composite material in a sensor preparation, what is all due to its unique two-dimensional structure and rich oxygencontaining functional groups [18]. Compared with the chemical method, the electrochemical reduction process forming electrochemically reduced graphene (ERGO) is quick, simple and nontoxic [19]. Likewise RGO, the surface charge of ERGO is partially negative in neutral electrolyte because of the oxygen-containing functional groups [20]. However, the molecular IMI is also of negative surface charge, what would bring electrostatic repulsion and hinder the adsorption and interaction between IMI and ERGO modified electrode.

To overcome this drawback, polypyrrole (PPy) was introduced into the electrode modification due to its positively charged nitrogen atoms [21,22]. The positively charged nitrogen atoms would provide interaction sites to enhance the interaction of IMI through electrostatic attraction. Besides, PPy shows remarkable sensing properties for its unique $\pi$-conjunction structure, perfect conductivity and abundant active sites. Moreover, the amine group ( $-\mathrm{NH}-$ ) on the pyrrole ring may lead to enhancement of the sensing performance [23,24]. However, as far as we know, there is no any report in the literature about electrochemical sensor for determination of imidacloprid based on PPy/ERGO composites.

In this study, a simple electropolymerization method has been used to prepare the glassy carbon electrode modified with PPy/ERGO composite for the detection of IMI. ERGO doped into PPy would not only compensate low conductivity of ERGO, but also remarkably promote the electron-transfer ability. CV and DPV techniques have been used to investigate electrochemical behavior of IMI at PPY/ERGO/GCE.

\section{Experimental}

\section{Reagents and apparatus}

IMI was purchased from Aladdin reagents (Shanghai Aladdin Biochemical Technology Co., Ltd.). All reagents were of analytical grade and used without further purification. Milli-Q water purifying system $(18 \mathrm{M} \Omega \mathrm{cm})$ was used for the preparation of deionized water which was used in all experiments. Citric acid/disodium hydrogen phosphate buffer solution (CPS) and sodium dihydrogen phosphate/disodium hydrogen phosphate buffer solution (PBS) were employed as electrolyte in the electrochemical characterizations. All experiments were performed at room temperature. 
Electrochemical characterizations were carried out with a three-electrode system by a CHI660D electrochemical workstation (Chenhua Instrument. Shanghai Co., Ltd., China). Bare and modified GCE were used as the working electrode, while a saturated calomel electrode (SCE) and a platinum wire electrode were employed as reference and auxiliary electrodes, respectively.

SEM (Japan Electron Optics Laboratory Co., Ltd) characterization was carried out by JEOL-6380LV, and Raman spectrum were conducted on Renishaw inVia Raman Microprobe using a $514.5 \mathrm{~nm}$ argon ion laser.

\section{Preparation of PPy/ERGO/GCE}

As reported in our previous works [25], GO was prepared from graphite powder via a modified Hummer's method [26]. The GCE was polished on the fur with an alumina powder/water and then ultrasonically cleaned in deionized water before modification. Before the polymerization, the homogenous GO-pyrrole dispersion was prepared with $1 \mathrm{~mL} \mathrm{GO}\left(5 \mathrm{mg} \mathrm{mL}^{-1}\right), 20 \mu \mathrm{L}$ pyrrole and $5 \mathrm{ml}$ water by 30 min ultrasonication. GO doped PPy was electro-polymerized onto the electrode surface through CV method in the GO-pyrrole dispersion with potential scanning between -0.2 and $1.0 \mathrm{~V}$ at $100 \mathrm{mV} \mathrm{s}^{-1}$. Then, GO was electrochemically reduced in $0.1 \mathrm{M} \mathrm{PBS}(\mathrm{pH} 7.0)$ by performing 5 cycles of $\mathrm{CV}$ in the potential range from 0 to $-1.7 \mathrm{~V}$ at $100 \mathrm{mV} \mathrm{s}^{-1}$. For comparison, PPy modified GCE (PPy/GCE) was prepared by the same procedure in acetonitrile solution containing $0.1 \mathrm{M} \mathrm{LiClO}_{4}$ and $0.1 \mathrm{M}$ pyrrole solution without GO. ERGO modified GCE (ERGO/GCE) was obtained from GO/GCE by the same procedure as described above.

\section{Electrochemical measurements}

Electrochemical behavior of IMI at various electrodes was characterized by CV and DPV methods in CPS (DPV conditions: potential increase, $0.004 \mathrm{~V}$; amplitude, $0.05 \mathrm{~V}$; pulse width, $0.05 \mathrm{~s}$; pulse interval, $0.2 \mathrm{~s}$ ). Electrochemical impedance spectroscopy (EIS) was used for the electron transfer property characterization of the modified electrodes in $0.1 \mathrm{M} \mathrm{KCl}$ solution with $5 \mathrm{mM}[\mathrm{Fe}(\mathrm{CN}) 6]^{3-/ 4-}$ (frequency range: $10^{6}-0.1 \mathrm{~Hz}$, amplitude: $5 \mathrm{mV}$ ). Possibly dissolved oxygen was expelled by nitrogen flow before every experiment.

\section{Results and discussion}

\section{Characterizations of PPy/ERGO/GCE}

The light yellow PPy/GO film was slowly deposited on the GCE through CV method as previously described. The electropolymerization CV curves at GCE in the GO-pyrrole dispersion are presented in Figure 1. As shown in Figure 1, during 10 cycles of electro-polymerization, the anodic current increased while the initial oxidation potential continuously shifted negatively, indicating the successful growth and thickening of the PPy/GO film.

The morphologies of the coated PPy and PPy/ERGO film on GCEs are shown in Figure 2 ( $A$ and B). It could be seen from the SEM images that PPy film deposited on the surface of the bare GCE has a relatively compact nanoparticle structure and some obvious ridges (Fig. 2A), while PPy/ERGO composite shows different morphology with PPy nanograins growing on the surface of ERGO sheets (Fig. 2B). PPy/ERGO sheets are randomly arranged on the surface of GCE to form a large amount of nanopores and micropores, which can definitely lead to a larger specific surface area (Fig. 2B). Favourable combination of PPy and ERGO and strong affinity of PPy/ERGO to the surface of GCE are beneficial to the diffusion of IMI from the solution to the electrode surface, and also facilitate fast exchange and transfer of electrons. 


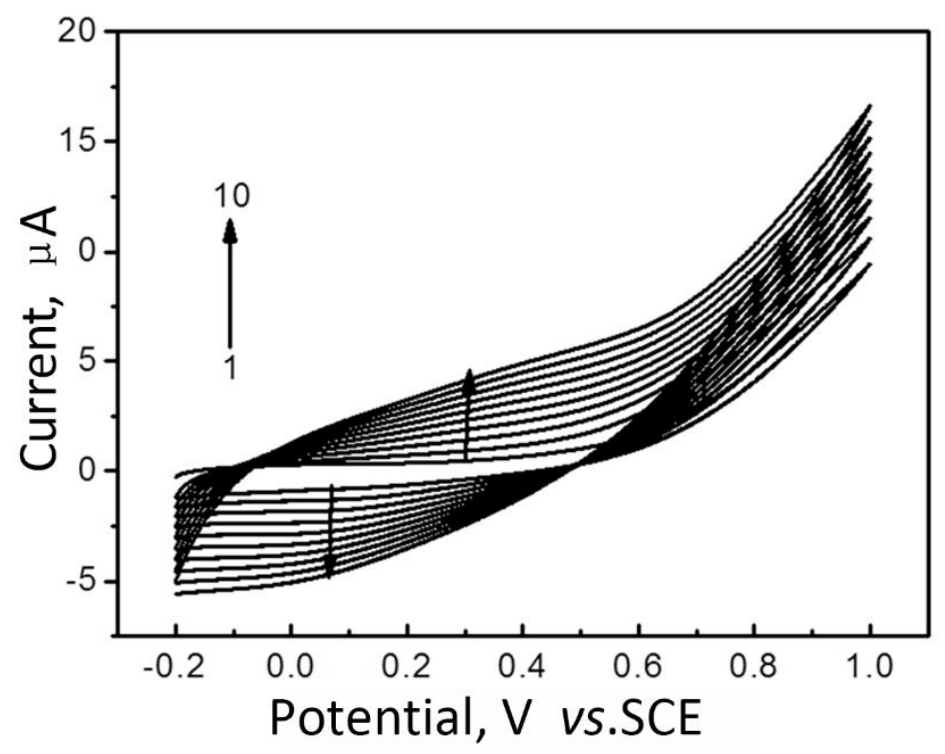

Figure 1. CV curves at GCE in the GO-pyrrole dispersion (20 $\mu \mathrm{L}$ pyrrole and $1 \mathrm{mg} \mathrm{mL}^{-1} \mathrm{GO}$ ) at a scan rate of $100 \mathrm{mV} \mathrm{s}^{-1}$ in the potential range between -0.2 and $1.0 \mathrm{~V}$
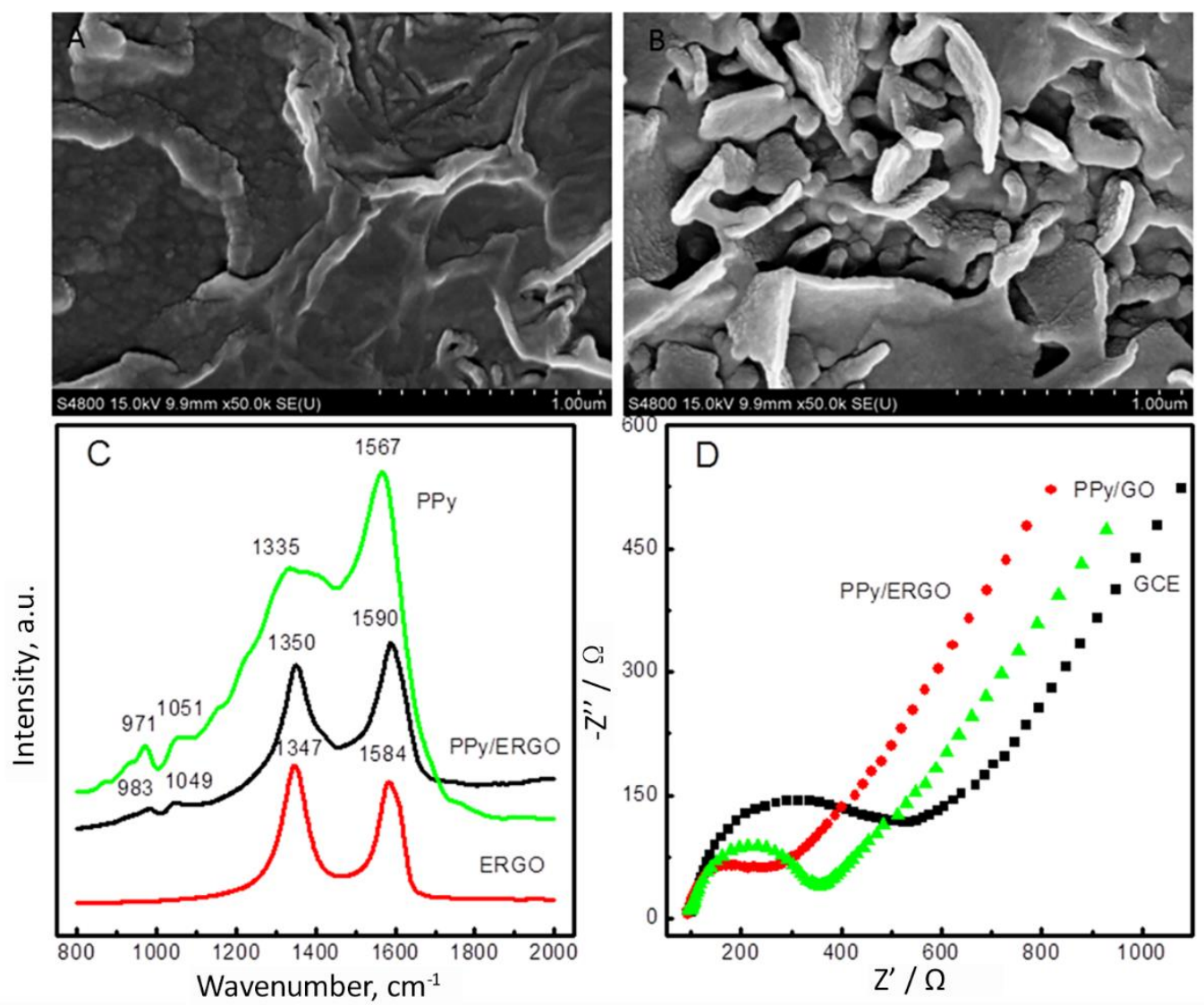

Figure 2. SEM of PPy (A) and PPY/ERGO (B); (C) Raman spectra of ERGO, PPy/ERGO and PPy;

(D) Nyquist plots in $0.1 \mathrm{M} \mathrm{KCl} \mathrm{containing} 5.0 \mathrm{mM}\left[\mathrm{Fe}(\mathrm{CN})_{6}\right]^{3-/ 4-}$ recorded at bare $\mathrm{GCE}$, $P P y / E R G O$ and PPy/GO modified GCEs (Initial potential: 0.18V)

Raman spectra of pure PPy, ERGO and PPy/ERGO are demonstrated in Figure 2C. For pure PPy, two strong peaks at 1335 and $1567 \mathrm{~cm}^{-1}$ represent the ring stretching mode and the backbone stretching mode of $C=C$ bonds in PPy, respectively. Peaks at 971 and $1051 \mathrm{~cm}^{-1}$ correspond to the ring deformation and the $\mathrm{C}-\mathrm{H}$ in-plane deformation of $\mathrm{PPy}$, respectively. As for ERGO, the characteristic $D$ and $G$ bands could be observed at 1347 and $1584 \mathrm{~cm}^{-1}$, respectively $[27,28]$. $D$ band 
exhibits the $\mathrm{sp}^{3}$ defects in the $\mathrm{sp}^{2}$-hybridized carbon network, while $\mathrm{G}$ band indicates doubly degenerate optical phonon of $E_{2 g}$ symmetry [29]. Compared with the spectra of PPy and ERGO, D and $G$ bands of PPy/ERGO shift to 1350 and $1590 \mathrm{~cm}^{-1}$ respectively, and the characteristic peaks of PPy appear at 983 and $1049 \mathrm{~cm}^{-1}$ respectively. These shifts indicate an interaction between PPy and ERGO sheets. Therefore, the Raman spectrum of PPy/ERGO composite reveals the presence of ERGO-doped PPy structures. This is in good agreement with Raman spectra results reported elsewhere [30].

\section{Electrochemical characteristics of PPy/ERGO modified GCE}

EIS was utilized for the characterization of electron transfer capability. Figure 2D shows the Nyquist plots of the bare GCE, PPy/GO/GCE and PPy/ERGO/GCE in $0.1 \mathrm{M} \mathrm{KCl}$ with $5 \mathrm{mM}$ $[\mathrm{Fe}(\mathrm{CN}) 6]^{3-/ 4-}$. Large well-defined semicircle can be observed at higher frequencies in the Nyquist plot of bare GCE, while the plot of PPy/GO/GCE shows a smaller semicircle, revealing the smaller interface impedance value of PPy/GO/GCE than GCE. This could be due to the fact that large surface area and high conductivity of PPy/GO promoted electron transfer. For the electrode modified with PPy/ERGO, a much smaller semicircle can be observed in Figure 2D, suggesting further decrease of interface impedance after reduction of PPy/GO. This indicates that ERGO plays a significant role in improving the electron-transfer process, what results in a superior electrochemical performance of PPy/ERGO/GCE over PPy/GO/GCE and GCE.

\section{Electrochemical behavior of IMI at PPy/ERGO modified GCE}

Electrochemical behavior of IMI at differently modified GCE were investigated with CV and DPV. Figure 3 shows CVs (A) and DPVs (B) at PPy/ERGO/GCE (a) and bare GCE (b) in CPS buffer solution containing $0.1 \mathrm{mM}$ IMI. For comparison, CVs and DPVs of PPy/ERGO/GCE in the blank CPS are also shown in Figures $3 \mathrm{~A}(\mathrm{c})$ and $\mathrm{B}(\mathrm{c})$ (scan rate: $100 \mathrm{mV} \mathrm{s}^{-1} ; \mathrm{pH}: 6.7$ ).
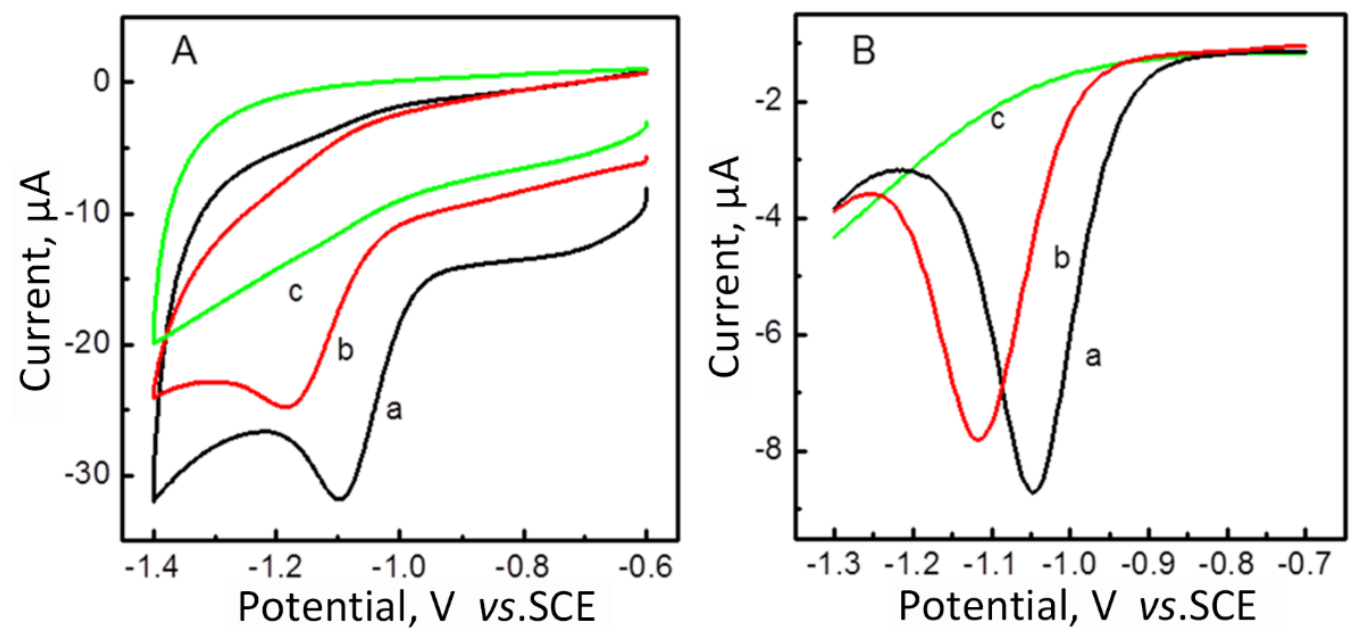

Figure 3. CVs (A) and DPVs (B) of PPy/ERGO/GCE $(\boldsymbol{a}, \boldsymbol{c})$ and bare GCE $(\boldsymbol{b})$ in presence $(\boldsymbol{a}, \boldsymbol{b})$ and absence (c) of $0.1 \mathrm{mM} \mathrm{IMI} \mathrm{in} \mathrm{CPS.} \mathrm{Scan} \mathrm{rate:} 100 \mathrm{mV} \mathrm{s}^{-1}, \mathrm{pH}$ : 6.7 .

As demonstrated in Figure 3, under given experimental conditions, no reduction peaks are observed for PPy/ERGO/GCE in the blank CPS (Fig. 3c). In presence of IMI, however, for both bare GCE (Fig. 3b) and PPy/ERGO/GCE (Fig. 3a), there is only one single reduction peak in CV and DPV, respectively. This suggests that nitro groups of IMI exhibit completely irreversible reduction process at the GCE and its modified electrodes. 
Irrespective of either CV or DPV applied, IMI reduction peak current values at PPy/ERGO/GCE are prominently higher than those at the bare GCE (Figs. 3a and 3b). At the same time, compared with the bare GCE, peak potential values are positively shifted at PPy/ERGO/GCE (from -1.18 to $-1.10 \mathrm{~V}$ in $\mathrm{CV}$; from -1.12 to $-1.03 \mathrm{~V}$ in DPV). Both suggest the satisfactory electrocatalytic activity of PPy/ERGO towards the reduction of IMI and high conductivity of the composite film. These electrosensing improvements can be attributed to a good combination of PPy with ERGO, and large surface area of the film with a porous structure.

\section{The effect of polymerization cycles}

The effect of the number $(2,5,10,15,20)$ of polymerization cycles on the determination of IMI at PPy/ERGO/GCE was optimized by CVs and DPVs in CPS pH 6.7 containing $0.1 \mathrm{mM} \mathrm{IMI}$. As shown in Figure 4 ( $A$ and $B$ ), the highest peak current value was obtained with ten polymerization cycles both in CV and DPV. With the further increase of the cycle number, the peak current values decreased apparently. The growing PPy/ERGO film would conversely hinder the electron transport as well as the analyte adsorption. Therefore, the optimized number of polymerization cycles is 10 .
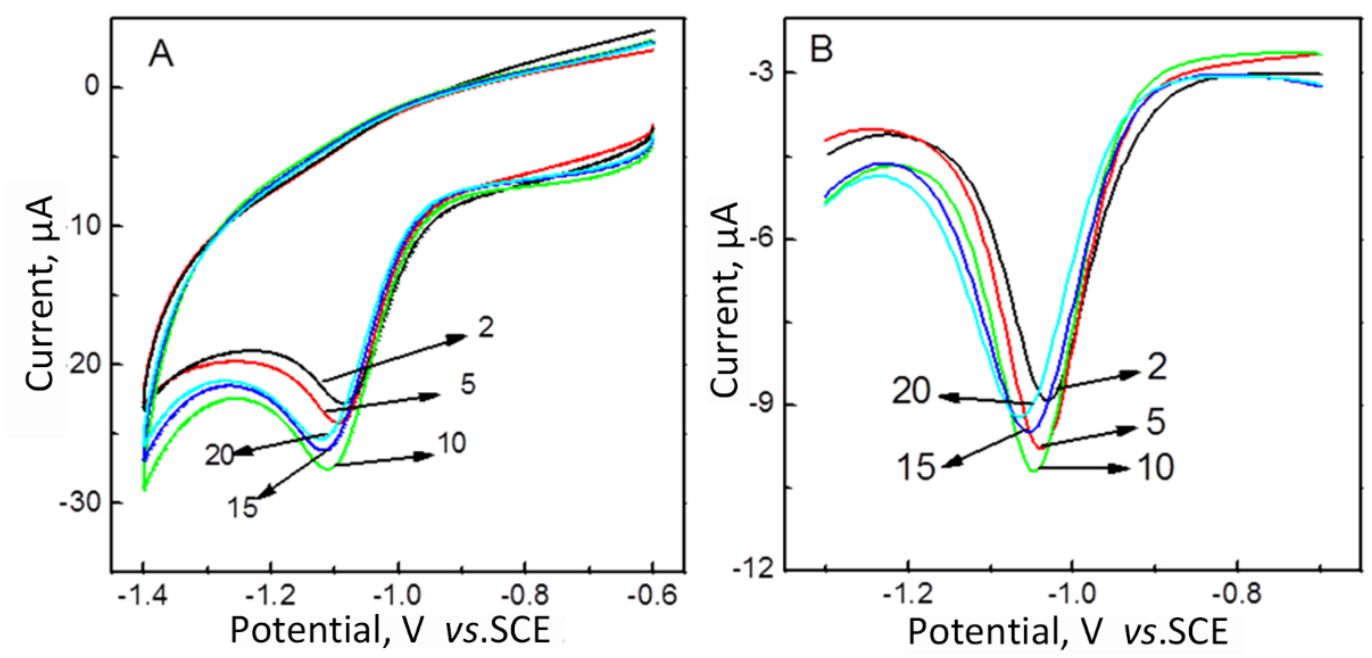

Figure 4. CVs of $0.1 \mathrm{mM} I \mathrm{MI}$ in $\mathrm{pH}=6.7 \mathrm{CPS}$ at $P P y / E R G O / G C E$ with various polymerization cycles $(2,5,10,15,20)$. Scan rate: $100 \mathrm{mV} \mathrm{s}^{-1}$.

\section{The effect of scan rate}

CVs of $0.1 \mathrm{mM} I \mathrm{MI}$ at PPy/ERGO/GCE obtained with the scan rate $(v)$ ranging from 10 to $200 \mathrm{mV} \mathrm{s}^{-1}$ in CPS pH 6.7 are presented in Figure 5A. As the scan rate increased, the reduction peak currents of IMI increased, while the peak potentials shifted negatively. The linear relationships between scan rate and the peak potential/current values are further depicted in Figure 5B-5C.

Figure $5 \mathrm{~B}$ demonstrates the linear relationship between the cathode peak current values of IMI and the square root of the scan rate $\left(v^{1 / 2}\right)$ within the range of 10 to $50 \mathrm{mV} \mathrm{s}^{-1}$. The obtained regression equation of $I_{\mathrm{pc}}(\mu \mathrm{A})$ vs. $v^{1 / 2}\left(\mathrm{mV} \mathrm{s}^{-1}\right)^{1 / 2}$ is:

$$
I_{\mathrm{pc}}=1.234 v^{1 / 2}+0.6415 \quad(\mathrm{R}=0.9993)
$$

Figure 5B and eqn. (1) indicate that the reduction reaction of IMI on PPy/ERGO/GCE is a typical diffusion-controlled process at low scan rates [31]. For high scan rates $\left(50-200 \mathrm{mV} \mathrm{s}^{-1}\right)$, the peak current values of IMI are linear with the scan rate (Fig. $5 \mathrm{C}$ ). The generated regression equation between $I_{\mathrm{pc}} / \mu \mathrm{A}$ and $v / \mathrm{mV} \mathrm{s}^{-1}$ is:

$$
I_{p c}=0.05043 v+7.089 \quad(R=0.9989)
$$


Figure $5 \mathrm{C}$ and eqn. (2) suggest that at the high scan rate, the reduction reaction of IMI is a typical adsorption-controlled process.

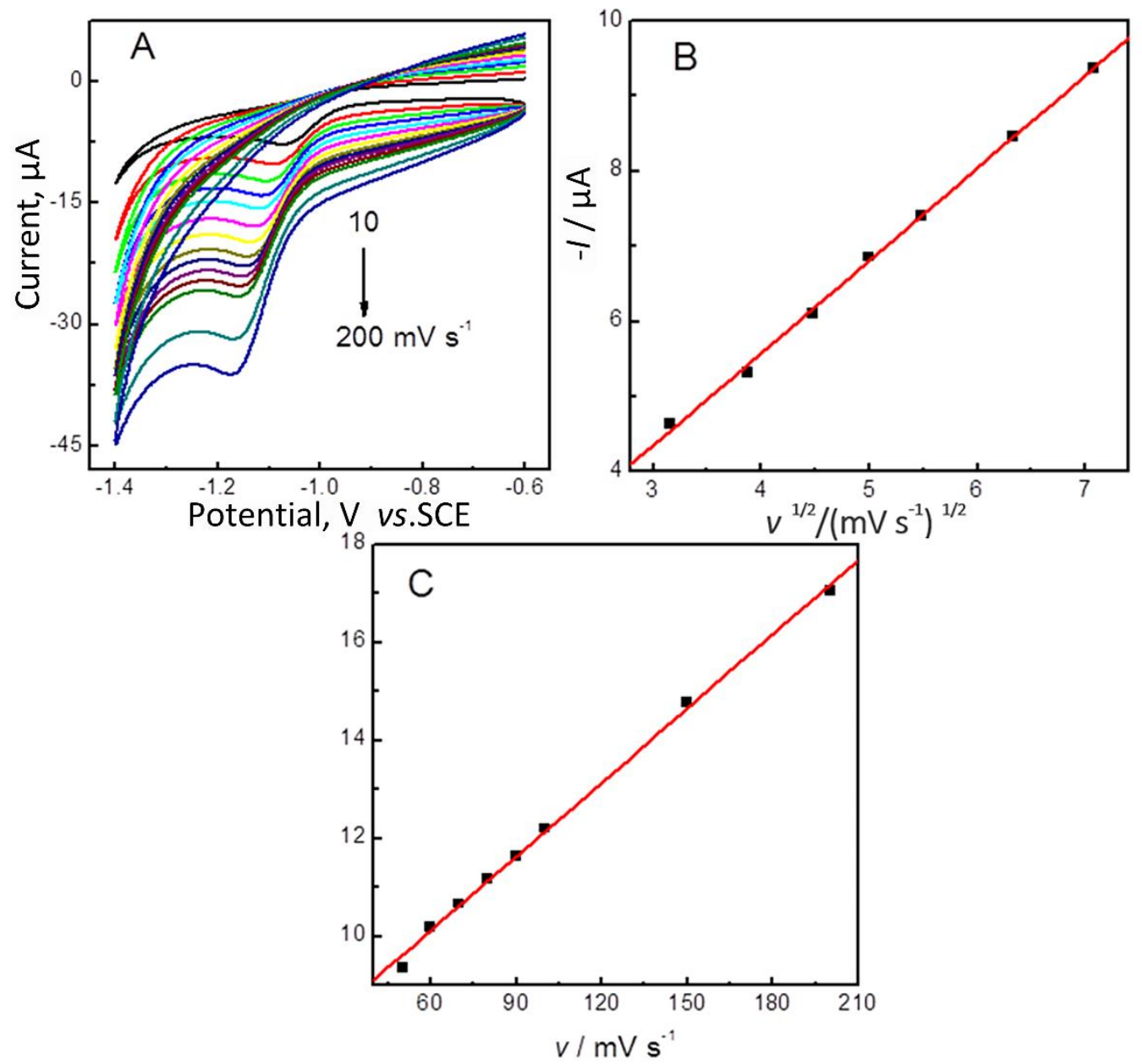

Figure 5. CVs acquired at a PPy/ERGO/GCE in $\mathrm{PH} 6.7 \mathrm{CPS}$ containing $0.1 \mathrm{mM} / \mathrm{MI}$ at different scan rate ( $\mathrm{V}$ ) of 10-200 $\mathrm{mV} \mathrm{s}^{-1}(\boldsymbol{A})$ and the influence of scan rate on peak current $(\boldsymbol{B}, \boldsymbol{C})$.

\section{The effect of $\mathrm{pH}$ on $\mathrm{CV}$ behavior of IMI}

Influence of $\mathrm{pH}$ on the CV behavior of $0.1 \mathrm{mM}$ IMI on PPy/ERGO/GCE was also studied and the results are presented in Figure 6. As $\mathrm{pH}$ was increased from 2.7 to 6.7, the reduction peak current values increased accordingly (Fig. 6A). When $\mathrm{pH}$ increased further up to 7.2, the reduction peak current value showed an obvious drop (Fig. 6B). Therefore, pH 6.7 was chosen as the optimized pH value in every electrochemical measurement. For the peak potential of the reduction peak, the change in potential was linear with the $\mathrm{pH}$ value (Fig. $6 \mathrm{C}$ ). The linear regression equation between $E_{\mathrm{pc}}(\mathrm{V})$ and $\mathrm{pH}$ was obtained as:

$$
E_{\mathrm{pc}}=-0.03078 \mathrm{pH}-0.9524 \quad(\mathrm{R}=0.9946)
$$

Figure $5 \mathrm{C}$ and eqn. (3) suggest participation of protons during the electro-catalyzed reduction reaction of IMI. The slope of the regression line is equal to $-2.303 m R T / \alpha n F$, in which, $\mathrm{m}$ and $\mathrm{n}$ are the number of protons and electrons, respectively. Therefore, $\mathrm{m} / \alpha \mathrm{n}$ was calculated as 0.52 (in the reaction, $\alpha=0.5$ and $n=4$ [31]). Therefore, the number of the involved protons $(\mathrm{m})$ is 1.2 , which is approximately equal to 1 . Thus, the electro-catalyzed reduction of IMI at PPy/ERGO/GCE is fourelectrons and one-proton process. 

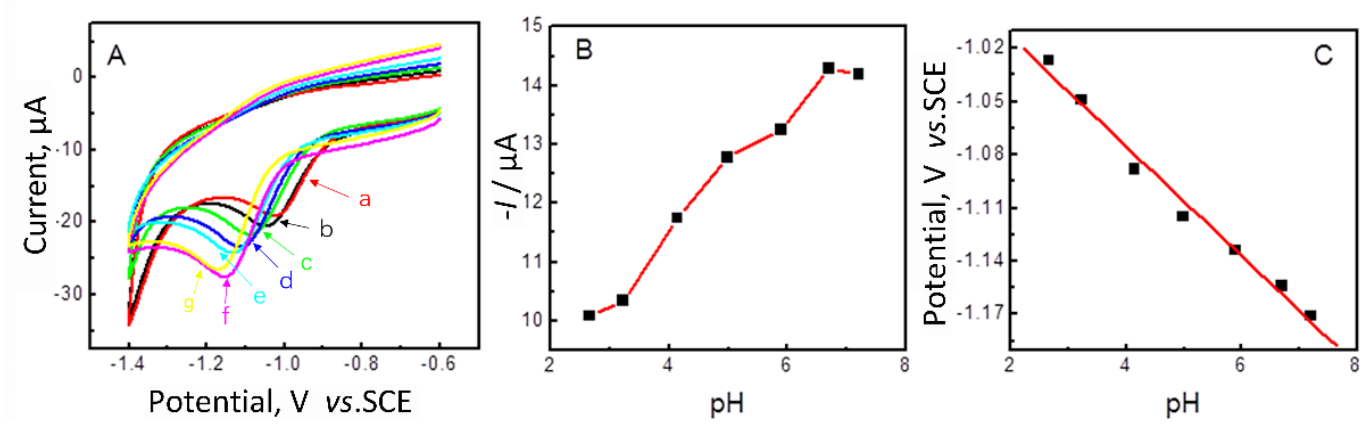

Figure 6. The influence of CPS $p H$ on the reduction peak current $(\boldsymbol{A}, \boldsymbol{B})$ and peak potential $(\boldsymbol{A}, \boldsymbol{C})$ of $0.1 \mathrm{mM} / \mathrm{Ml}$ at an optimized PPy/ERGO/GCE.

\section{DPV determinations of IMI}

Enhancement of the electrochemical signal observed at PPy/ERGO/GCE compared with the bare GCE and PPy modified GCE, makes PPy/ERGO to be an attractive electrocatalytic candidate for the determination of IMI. DPV technique, that is usually considered to have a higher sensitivity than CV, was utilized here for the quantitative determination of IMI under optimized conditions. Figure 7A shows well-defined DPV reduction peaks at PPy/ERGO/ERGO in CPS, $\mathrm{pH} 6.7$ containing different concentrations of IMI. Due to weak adsorption of IMI on the surface of the modified electrode, the reduction peak current values increased when IMI concentration gradually increased from $1 \mu \mathrm{M}$ to $60 \mu \mathrm{M}$. Moreover, the peak current values were found proportional to the concentration of IMI. Finally, two linear relationships between DPV peak current value and IMI concentration were extracted, covering two linear ranges of 1-10 $\mu \mathrm{M}$ and $10-60 \mu \mathrm{M}$, respectively. The corresponding linear equations between $I_{\mathrm{pc}}(\mu \mathrm{A})$ and $c(\mu \mathrm{M})$ are:

$$
\begin{array}{ll}
I_{\mathrm{pc}}=0.1265 c+0.3279 & (\mathrm{R}=0.9996) \\
I_{\mathrm{pc}}=0.03983 c+1.192 & (\mathrm{R}=0.9996)
\end{array}
$$

The low detection limits are by eqns. (4) and (5) determined as $0.18 \mu \mathrm{M}$ and $1.2 \mu \mathrm{M}(\mathrm{S} / \mathrm{N}=3$ ), respectively. As compared with other literature work presented in Table 1, here proposed DPV sensing method with PPy/ERGO/GCE shows relative low detection limit, demonstrating thus clearly high potential of this electrode for sensitive determination of IMI.

It is apparent from Figure 7 and equations (4) and (5) that there is a significant change in slopes of linear lines at a single specific concentration, and the slope at high concentrations is much smaller than at low concentrations. We speculate that this phenomenon is due to the poor conductivity of IMI, and the accumulation of IMI at high concentrations hinders the exchange of electrons between itself and the electrodes. In this way, the sensitivity of the sensor is reduced, so the slope at high concentration is lower than that at low concentration.

Table 1 Comparison of electrochemical sensors for IMI

\begin{tabular}{cccc}
\hline Electrode & Method & LOD, $\mu \mathrm{M}$ & References \\
\hline AgNDs/GNs/GCE & DPV & 0.81 & {$[32]$} \\
nAgnf/nTiO2nf/GCE & $\mathrm{CV}$ & 0.63 & {$[10]$} \\
Poly(o-phenylenediamine)-rGO/GCE & $\mathrm{LSV}$ & 0.40 & {$[33]$} \\
poly(carbazole)/chemically reduced graphene & $\mathrm{CV}$ & 0.22 & {$[25]$} \\
oxide/GCE & $\mathrm{DPV}$ & 0.44 & {$[25]$} \\
$\beta$-cyclodextrin polymer/GCE & $\mathrm{CV}$ & 0.10 & {$[34]$} \\
GO/GCE & $\mathrm{CV}$ & 0.36 & {$[35]$} \\
PPy/ERGO/GCE & $\mathrm{DPV}$ & 0.18 & This work \\
\hline
\end{tabular}



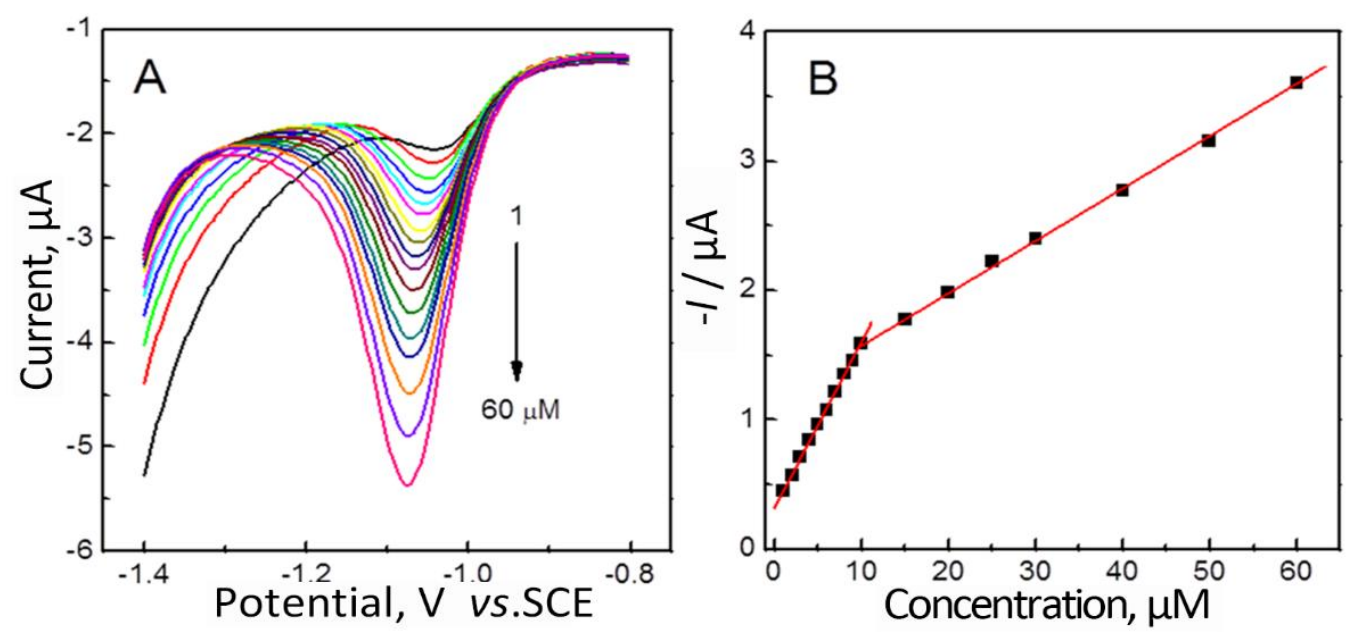

Figure 7. DPVs (A) of IMI with different concentrations (1-60 $\mu \mathrm{M})$ at PPy/ERGO/GCE in CPS solution ( $p H$ 6.7). (B) Relationship between the DPV peak current and IMI concentration

\section{Reproducibility, repeatability and interference}

For the sake of demonstrating the accuracy and practicability of the method proposed, the reproducibility of the as-fabricated PPy/ERGO/GCE was investigated by comparing the peak current of ten experiments performed successively in CPS containing $0.1 \mathrm{mM}$ IMI. The relative standard deviation (RSD) was $2.2 \%$, which reflected that this method has the outstanding reproducibility. Under optimal conditions, after storing the electrode at room temperature for seven days, we did not notice any obvious change in the peak current. The interference test was performed in the presence of the inorganic ions and some pesticides, for instance $0.1 \mathrm{M} \mathrm{K}^{+}, \mathrm{Na}^{+}, \mathrm{Ce}^{2+}, \mathrm{Zn}^{2+}, \mathrm{Pb}^{2+}, \mathrm{Cl}^{-}, \mathrm{NO}_{3}{ }^{-}, \mathrm{SO}_{4}{ }^{2-}$, carbendazim, trichlorfon and glyphosate. The reduction signal did not change considerably, indicating that all these inorganic ions and pesticides have no effect on the determination of IMI and illustrating that the electrochemical sensor based on PPy/ERGO/GCE exhibits high selectivity.

\section{Conclusion}

In this work, PPy/ERGO modified GCE was successfully used as a platform for electrochemical sensing of imidacloprid insecticides. The recorded CVs showed a remarkably enhanced electrochemical response of IMI at PPy/ERGO/GCE. Under the optimized conditions and using DPV technique, two linear ranges for IMI determination were obtained between 1.0-10 $\mu \mathrm{M}$ and $10-60.0 \mu \mathrm{M}$ respectively. The limit of detection was $0.18 \mu \mathrm{M}$. These results indicated that PPy/ERGO is good functional material, suitable for sensing of IMI with high sensitivity, selectivity, and stability. Furthermore, here proposed sensing method is rapid, reliable and easy for manipulation. Therefore, the proposed strategy based on a PPy/ERGO modified electrode could be a promising approach for quantitative detection of imidacloprid insecticide.

Acknowledgements: The work was supported by the National Natural Science Foundation of China (Nos. 51572127, 21576138), Program for NCET-12-0629, Ph.D. Program Foundation of Ministry of Education of China (No.20133219110018), Six Major Talent Summit (XNY-011), Natural Science Foundation of Jiangsu Province (BK20160828), Postdoctoral Science Foundation (1501016B) and PAPD of Jiangsu Province, and the program for Science and Technology Innovative Research Team in Universities of Jiangsu Province, China.

\section{References}

[1] A. Brandt, A. Gorenflo, R. Siede, M. Meixner, R. Büchler, Journal of Insect Physiology 86 (2016) 40-47

[2] R. Ramachandran, V. Mani, S. M. Chen, G. G. Kumar, M. Govindasamy, International Journal of Electrochemical Science 10 (2015) 691-700 
[3] K. L. Klarich, N. C. Pflug, E. M. Dewald, M. L. Hladik, D. W. Kolpin, D. M. Cwiertny, G. H. Lefevre, Environmental Science \& Technology 4 (2017) 168-173

[4] H. Obana, M. Okihashi, K. Akutsu, Y. Kitagawa, S. Hori, Journal of Agricultural and Food Chemistry 50 (2002) 4464-4467

[5] A. Niaz, R. A. Sial, M. Yaseen, G. A. Mand, M. H. Javed, E. Ahmad, R. Ahmad, M. Rahim, Journal of Animal \& Plant Sciences 26 (2016) 170-176

[6] J. Cheng, M. L. Wang, S. H. Zhu, J. W. Wen, L. Ding, L. B. Wang, Journal of Food Safety \& Quality 2016-1 (2016) 131-137

[7] M. A. Farooqi, MansoorulHasan, M. A. Sabri, N. Javed, Pakistan Journal of Zoology 47 (2015) 965-970

[8] W. Liang, J. Wang, X. Zang, W. Dong, C. Wang, Z. Wang, Journal of Chromatography A 1491 (2017) 9

[9] V. Guzsvany, Z. Papp, J. Zbiljic, O. Vajdle, M. Rodic, Molecules 16 (2011) 4451-4466

[10] A. Kumaravel, M. Chandrasekaran, Sensors and Actuators B: Chemical 158 (2011) 319-326

[11] S. Stankovich, D. A. Dikin, G. H. B. Dommett, K. M. Kohlhaas, E. J. Zimney, E. A. Stach, R. D. Piner, S. T. Nguyen, R. S. Ruoff, Nature 442 (2006) 282

[12] C. G. Lin, J. Hu, Y. F. Song, Advances in Inorganic Chemistry 8 (2017) 776-789

[13] V. Georgakilas, J. N. Tiwari, K. C. Kemp, J. A. Perman, A. B. Bourlinos, K. S. Kim, R. Zboril, Chemical Reviews 116 (2016) 5464

[14] T. Szabó, E. Tombácz, E. Illés, I. Dékány, Carbon 44 (2006) 537-545

[15] G. Yang, G. Zhang, P. Sheng, F. Sun, W. Xu, D. Zhang, Journal of Materials Chemistry 22 (2012) 43914395

[16] L. G. Guex, B. Sacchi, K. F. Peuvot, R. L. Andersson, A. M. Pourrahimi, V. Ström, S. Farris, R. T. Olsson, Nanoscale 9 (2017) 9562-9571

[17] M. A. Raj, S. A. John, Journal of Physical Chemistry C 117 (2016) 4326

[18] C. I. L. Justino, A. R. Gomes, A. C. Freitas, A. C. Duarte, T. A. P. Rocha-Santos, TRAC Trends in Analytical Chemistry 91 (2017) 53-66

[19] M. Gao, Y. Xu, X. Wang, Y. Sang, S. Wang, Electroanalysis 28 (2016) 1377-1382

[20] S. Y. Toh, K. S. Loh, S. K. Kamarudin, R. W. D. Wan, Chemical Engineering Journal 251 (2014) $422-434$

[21] S. C. Rasmussen, Bulletin for the History of Chemistry 40 (2015) 45-55

[22] K. L. Konkol, R. L. Schwiderski, S. C. Rasmussen, Materials 9 (2016) 404

[23] A. Turco, S. Corvaglia, E. Mazzotta, Biosensors \& Bioelectronics 63 (2015) 240-247

[24] Y. Li, H. Ban, M. Yang, Sensors \& Actuators B: Chemical 224 (2016) 449-457

[25] W. Lei, Q. Wu, W. Si, Z. Gu, Y. Zhang, J. Deng, Q. Hao, Sensors and Actuators B: Chemical 183 (2013) 102-109

[26] H. Wang, Q. Hao, X. Yang, L. Lu, X. Wang, Nanoscale 2 (2010) 2164-2170

[27] S. Dhibar, C. K. Das, Journal of Applied Polymer Science 134 (2017) 16

[28] R. Rong, H. Zhao, X. Gan, S. Chen, X. Quan, Nano 12 (2016) 1750008

[29] X. Sheng, D. Xie, W. Cai, X. Zhang, Z. Li, H. Zhang, Industrial \& Engineering Chemistry Research 54 (2015) 649-658

[30] C. Bora, S. K. Dolui, Polymer 53 (2012) 923-932

[31] W. Si, W. Lei, Y. Zhang, M. Xia, F. Wang, Q. Hao, Electrochimica Acta 85 (2012) 295-301

[32] M. R. Majidi, S. Ghaderi, Journal of Electroanalytical Chemistry 792 (2017) 46-53

[33] L. Kong, X. Jiang, Y. Zeng, T. Zhou, G. Shi, Sensors and Actuators B: Chemical 185 (2013) 424-431

[34] M. Chen, Y. Meng, W. Zhang, J. Zhou, J. Xie, G. Diao, Electrochimica Acta 108 (2013) 1-9

[35] W. Lei, Z. Han, W. Si, Q. Hao, Y. Zhang, M. Xia, F. Wang, ChemElectroChem 1 (2014) 1063-1067

(C)2019 by the authors; licensee IAPC, Zagreb, Croatia. This article is an open-access article distributed under the terms and conditions of the Creative Commons Attribution license (http://creativecommons. org/licenses/by/4.0/) 